\title{
Effectiveness of national food-based dietary guidelines in reducing cardiometabolic risk factors: a systematic review of randomised controlled trials
}

\author{
F. Deli ${ }^{1}$, A.M. Pinto ${ }^{1}$ and W.L. Hall ${ }^{1}$ \\ ${ }^{1}$ Department of Nutritional Sciences, School of Life Course Sciences, King's College London, London, UK
}

Food-based dietary guidelines (FBDGs) are evidence-based dietary recommendations that advise the general population on the adoption of healthy dietary patterns to help prevent non-communicable diseases. This is especially relevant in the context of cardiometabolic diseases, which are major causes of death and disability in the world ${ }^{(1)}$. However, a systematic evaluation of dietary intervention studies investigating the impact of FBDGs on cardiometabolic health has not yet been conducted.

The aim of this systematic review was to critically analyse the evidence from randomised controlled trials (RCTs) that FBDGs beneficially impact cardiometabolic risk factors in healthy and at- risk individuals aged $>18$ years.

A systematic review was conducted according to the PRISMA guidelines ${ }^{(2)}$ (protocol registration DOI: 10.17605/OSF.IO/JKBV9). The following online databases were searched up to June 2021 using a comprehensive search strategy: Embase, MEDLINE, Web of Science and CENTRAL. Studies were screened for inclusion based on eligibility criteria (briefly, subjects free of diagnosed disease and not on weight loss intervention, intervention followed national FBDG, outcomes included CVD risk factors or CVD events), and data were extracted following a pre-specified plan. Primary outcomes were systolic blood pressure, diastolic blood pressure, TC:HDL-C ratio and LDL-C:HDL-C ratio. The effects of the interventions were reported as mean difference (MD) and 95\% confidence interval $(95 \% \mathrm{CI})$ and results were summarised narratively. Risk of bias was assessed according to version 2 of the Cochrane risk of bias tool ${ }^{(3)}$.

Nine RCTs assessing the impact of food provision or dietary advice to follow FBDGs on cardiometabolic risk factors were identified and included in the review. Four out of eight studies measuring blood pressure found a significant reduction in both systolic and diastolic blood pressure. Five out of eight studies assessing the lipid profile found significant improvements in some lipid components, but results were mixed for each component. One study investigated vascular function and found a significant reduction in arterial stiffness but no effect on endothelial function assessed by flow-mediated dilation. Seven studies measured glycaemic biomarkers but none reported significant effects on plasma glucose, plasma insulin and insulin sensitivity. Five out of eight studies measuring anthropometry found a significant reduction in BMI, waist circumference, body fat percentage and body weight. One out of four studies measuring inflammatory biomarkers found a significant reduction in high-sensitivity C-reactive protein. Risk of bias was rated as low for one study, moderate for six studies and high for two studies, primarily due to issues with blinding, methods used to measure adherence to the diets, and high rates of withdrawal and loss to follow-up.

In conclusion, adherence to national FBDGs can improve certain risk factors for cardiometabolic diseases. However, more RCTs using standardised study designs are needed to better calculate the effect size of FBDGs.

\section{Acknowledgments}

I would like to express my gratitude to Dr Wendy Hall and Dr Ana Margarida Pinto for providing ongoing support and advice throughout this project. I would also like to thank Dr Christine Baldwin for providing feedback on the search strategy.

\section{References}

1. Roth GA, Abate D, Abate KH et al. (2017) The Lancet 392, 1736-1788.

2. Page MJ, McKenzie JE, Bossuyt PM et al. (2021) BMJ 372, n71.

3. Sterne JA, Savović J, Page MJ et al. (2019) BMJ 366, 14898. 